\title{
Echocardiographic changes in elderly patients with heart failure with reduced ejection fraction after sacubitril-valsartan treatment
}

\author{
Wei Gu${ }^{1 \#}$, Chuangye $\mathrm{Xu}^{2 \#}$, Zhao $\mathrm{Li}^{1}$, Zhi-Zhong $\mathrm{Li}^{1}$ \\ ${ }^{1}$ Department of Cardiology, Beijing Anzhen Hospital, Capital Medical University and Beijing Institute of Heart Lung and Blood Vessel Diseases, \\ Beijing, China; ${ }^{2}$ School of Medicine, Southern University of Science and Technology, Shenzhen, China \\ Contributions: (I) Conception and design: W Gu, C Xu, ZZ Li; (II) Administrative support: C Xu, Z Li; (III) Provision of study materials or patients: \\ W Gu, Z Li; (IV) Collection and assembly of data: C Xu, Z Li; (V) Data analysis and interpretation: W Gu, C Xu, Z Li; (VI) Manuscript writing: All \\ authors; (VII) Final approval of manuscript: All authors. \\ "These authors contributed equally to this work. \\ Correspondence to: Zhi-Zhong Li. Department of Cardiology, Beijing Anzhen Hospital, No. 2 Anzhen Rd, Chaoyang District, Beijing 100029, China. \\ Email: lzzeagle@sina.com.
}

Background: To observe the changes of cardiac structure and function in elderly patients with heart failure with reduced ejection fraction (HFrEF) after taking Sacubitril-Valsartan for 6 months.

Methods: Elderly patients with HFrEF hospitalized in Beijing Anzhen Hospital from May 2019 to May 2020 were enrolled continuously in the single-center, retrospective, cohort study. Patients' Echocardiographs were examined for the evaluation of their cardiac condition. The primary outcomes were changes in cardiac function and structure at the sixth month after discharge, including left ventricular ejection fraction (LVEF), left ventricular end-diastolic diameter (LVEDD), left ventricular end-systolic diameter (LVESD), left atrial diameter (LAD), interventricular septum thickness (IVST), left ventricular posterior wall thickness (LVPWT) and left ventricular mass index (LVMI).

Results: A total of 336 elderly patients with HFrEF were enrolled in this study, with an average age of 69.8 years, including 268 males (79.8\%). Compared to the admission levels, the LVEF after taking SacubitrilValsartan for 6 months was markedly improved (48.49\% vs. 39.07\%, P<0.01), while the LVEDD (54.70 vs. $59.97 \mathrm{~mm}, \mathrm{P}<0.01$ ), LVESD (40.59 vs. $47.59 \mathrm{~mm}, \mathrm{P}<0.01$ ), LAD (48.59 vs. $52.45 \mathrm{~mm}, \mathrm{P}<0.01$ ) and LVMI (105.16 vs. $125.20 \mathrm{~g} / \mathrm{m}^{2}, \mathrm{P}<0.01$ ) decreased. Similar results were obtained in the subgroups of patients who were diagnosed with HFrEF on admission. In men, NHYA II and NHYA III subgroups, cardiac function improved significantly.

Conclusions: Sacubitril-Valsartan can improve the cardiac function and structure of elderly patients with HFrEF.

Keywords: Sacubitril-valsartan; cardiac structure; cardiac function; heart failure with reduced ejection fraction (HFrEF); echocardiographic change

Submitted Jun 07, 2021. Accepted for publication Sep 12, 2021.

doi: $10.21037 / \mathrm{cdt}-21-355$

View this article at: https://dx.doi.org/10.21037/cdt-21-355

\section{Introduction}

Heart failure (HF) is a group of syndromes caused by various cardiac structural and functional diseases, in which ventricular filling and/or ejection function are often impaired. This means that HF is the terminal state of various cardiac diseases. According to the latest epidemiological studies, there are about 4 million patients with chronic HF in China, and the number continues to rise $(1,2)$. In addition, the prevalence, hospitalization and mortality rate increase with age. For a long time, the Gold Triangle therapy, including renin-angiotensin system inhibitor, $\beta$-receptor agonist and aldosterone receptor 
antagonist, was the leading treatment scheme for HF, especially for heart failure with reduced ejection fraction (HFrEF). However, its efficiency varies from person to person, and some patients can only get noticeable results after taking the drugs for a long period of time. In recent years, Sacubitril-Valsartan has provided a novel option for treating chronic HF by replacing renin-angiotensin system inhibitor to form a new Golden Triangle scheme, which shows excellent prospects in controlling HF symptoms and reducing hospitalization and mortality rate.

Sacubitril-Valsartan can not only inhibit the angiotensin receptor and block renin-angiotensin-aldosterone (RAAS), but also inhibit enkephalinase and increase the content of brain natriuretic peptide. Blocking the RAAS system plays an important role in the treatment of HF, which has been proven by many studies. Inhibition of enkephalinase can increase natriuretic peptide and bradykinin to lower blood pressure, dilate blood vessels and improve myocardial remodeling. At the same time, enkephalinase inhibition can inhibit the reabsorption of sodium by nephrons and increase the amount of urine sodium and urine output $(3,4)$. Many large-scale studies, such as the classic PARADIGMHF study, have found that Sacubitril-Valsartan could reduce cardiovascular deaths, hospitalization rates and allcause mortality of patients with HFrEF, and could prevent $\mathrm{HF}$ from deteriorating and significantly improve the quality of life (5). Meanwhile, other studies have shown that Sacubitril-Valsartan can improve cardiac structure by protecting damaged myocardium and reversing myocardial remodeling (6).

Although Sacubitril-Valsartan has shown preliminary results in HF treatment, there are no detailed reports on the echocardiography changes in elderly patients with HFrEF. Therefore, we intend to retrospectively analyze the elderly patients with HFrEF treated with Sacubitril-Valsartan to explore the effect of reserving myocardial remodeling by evaluating the changes of cardiac structure and function through echocardiography.

We present the following article in accordance with the STROBE reporting checklist (available at https://dx.doi. org/10.21037/cdt-21-355).

\section{Methods}

\section{Study population}

Elderly patients with HF hospitalized in Beijing Anzhen Hospital from May 2019 to May 2020 were continuously enrolled in the single center, retrospective, cohort study. The inclusion criteria were: age $\geq 60$ and $\leq 80$ years old; left ventricular ejection fraction (LVEF) $\leq 40 \%$ or HFrEF patients with NYHA II, III and IV; taking SacubitrilValsartan during hospitalization. Echocardiographic examination on these patients was performed on admission and at the sixth month after discharge. Among all participants, the diagnostic criteria of HFrEF referred to Chinese guidelines (2). The exclusion criteria included: acute HF patients; contraindicated to Sacubitril-Valsartan; seriously infected or malnourished; patients with malignant tumor and other serious internal and surgical diseases. This study complied with the Declaration of Helsinki (as revised in 2013), local regulations, and was approved by Beijing Anzhen Hospital's Ethics Committee (2021090X), and individual consent for this retrospective analysis was waived.

\section{Outcome measures}

The baseline data was collected comprehensively, including age, sex, height, weight, systolic blood pressure (SBP), diastolic blood pressure (DBP), heart rate (HR), B-type natriuretic peptide (BNP), history of hypertension, diabetes, percutaneous coronary intervention (PCI) or coronary artery bypass graft (CABG), previous medication, initiation dose of Sacubitril-Valsartan, creatinine, estimated glomerular filtration rate (eGFR) and serum kalium. LVEF, left ventricular end-diastolic diameter (LVEDD), left ventricular end-systolic diameter (LVESD), left atrial diameter (LAD), interventricular septum thickness (IVST) and left ventricular posterior wall thickness (LVPWT) on admission were obtained from echocardiography. left ventricular mass index (LVMI) was calculated according to the formula provided by Devereux RB (7).

The primary outcomes of this study were changes in cardiac structure and function sixth months after discharge, including LVEF, LVEDD, LVESD, LAD, IVST, LVPWT and LVMI. If echocardiography was examined at the third month for participants, the related data would be collected. In subgroup analysis, patients diagnosed as HFrEF on admission were analyzed separately. Then, the changes in cardiac function and structure in subgroups of different gender and NYHA classes were evaluated.

\section{Statistical analysis}

SPSS 24.0 software was used for data analysis. The measurement data conforming to normal distribution was 
Table 1 Baseline characteristics of the participants ( $n=336)$

\begin{tabular}{|c|c|}
\hline Parameter & Number \\
\hline Age, y & 69.8 \\
\hline Males (\%) & 79.8 \\
\hline Height, cm & 168.2 \\
\hline Weight, kg & 74.3 \\
\hline \multicolumn{2}{|l|}{ Baseline vital signs } \\
\hline Systolic blood pressure, $\mathrm{mmHg}$ & 124.8 \\
\hline Diastolic blood pressure, $\mathrm{mmHg}$ & 76.0 \\
\hline Heart rate, beats/min & 74.2 \\
\hline B-type natriuretic peptide, $\mathrm{pg} / \mathrm{mL}$ & 562.8 \\
\hline Ischemic etiology for HF (\%) & 68.5 \\
\hline \multicolumn{2}{|l|}{ NYHA symptom severity class (\%) } \\
\hline II & 58.6 \\
\hline III & 37.2 \\
\hline IV & 4.2 \\
\hline \multicolumn{2}{|l|}{ Medical History (\%) } \\
\hline Hypertension & 64.9 \\
\hline Diabetes & 37.8 \\
\hline $\mathrm{PCl}$ or $\mathrm{CABG}$ & 22.3 \\
\hline \multicolumn{2}{|l|}{ Previous medication (\%) } \\
\hline ACEI or ARB & 33.3 \\
\hline Diuretics & 68.5 \\
\hline Digoxin & 19.6 \\
\hline Beta-blocker & 88.7 \\
\hline Spironolactone & 68.5 \\
\hline Creatinine, $\mu \mathrm{mol} / \mathrm{L}$ & 104.8 \\
\hline eGFR, mL/min/1.73 $\mathrm{m}^{2}$ & 76.6 \\
\hline $\mathrm{K}+, \mathrm{mmol} / \mathrm{L}$ & 4.16 \\
\hline
\end{tabular}

NYHA, New York Heart Association; PCl, percutaneous coronary intervention; CABG, coronary artery bypass graft; ACEI, angiotensin-converting enzyme inhibitor; ARB, angiotensin II receptor blocker; eGFR, estimated glomerular filtration rate.

indicated by mean \pm standard deviation $(\mathrm{SD})$, and that not conforming to normal distribution was indicated by median (quartile). The Kolmogorov-Smirnov test was used to test for normal distribution. Counting data and classification variables were given as frequency or percentage (\%). Paired $t$-test was used to compare the data at the sixth month with data at the baseline for the primary outcomes. Sample size was calculated by tests for paired means using PASS version 15.0. For patients who underwent ultrasound examination at the third and sixth month, the echocardiography data (at baseline, the third month and the sixth month) was compared by variance analysis based on repeated measurement data. Meanwhile, the mixed effect model was used to analyze the change of echocardiography data at the third and sixth month with those at baseline after adjusting for confounding factors including age, sex, height, weight, SBP, DBP, HR, BNP, NYHA symptom severity class, medical history, previous medication, creatinine and eGFR. All statistical tests were conducted by bilateral test, and the difference was statistically significant with $\mathrm{P}$ value $<0.05$.

\section{Results}

\section{Baseline data}

A total of 336 elderly patients with HFrEF were enrolled in this study, including 268 males (79.8\%), with an average age of 69.8 years, SBP and DBP of 124.8 and $76.0 \mathrm{mmHg}$, BNP of $562.8 \mathrm{pg} / \mathrm{mL}$ and creatinine of $104.8 \mu \mathrm{mol} / \mathrm{L}$. Among all patients, ischemic cardiomyopathy accounted for a large proportion (230 cases, $68.5 \%$ ), while the others were dilated cardiomyopathy and hypertensive or rheumatic heart disease (106 cases, $31.5 \%$ ). At enrollment, participants had either NYHA II (197 cases, 58.6\%) or III (125 cases, $37.2 \%)$ or IV (14 cases, $4.2 \%)$ symptoms. Among all the patients, 218 cases were complicated with hypertension (64.9\%), 127 cases $(37.8 \%)$ with diabetes, and 75 cases $(22.3 \%)$ with history of coronary stent implantation or bypass grafting. Previous medication conditions: ACEI or ARB drugs (33.3\%), diuretics (68.5\%), digoxin (19.6\%), beta-blocker $(88.7 \%)$, spironolactone $(68.5 \%)$. Baseline characteristics of the participants were shown in Table 1.

Dosage of Sacubitril-Valsartan was adjusted according to the improvement of blood pressure and HF symptoms. Among all patients, the initial twice daily doses included $25 \mathrm{mg}$ (88 cases, 26.2\%), $50 \mathrm{mg}$ (182 cases, $54.2 \%$ ), $75 \mathrm{mg}$ (5 cases, $1.5 \%$ ) and $100 \mathrm{mg}$ (61 cases, $18.2 \%)$. During titration, 27 cases $(8.0 \%)$ changed the number of taking medicine due to cost or other reasons; 15 cases $(4.5 \%)$ reduced to $50 \mathrm{mg}$ twice daily from high dose SacubitrilValsartan because of intolerance to hypotension; 130 cases (38.7\%) maintained the initial dosage; a total of 164 cases (48.8\%) increased the dosage. After titration, 45 cases (13.4\%) finally reached the target dose of $200 \mathrm{mg}$ twice daily. 


\section{Follow-up of the overall population}

Echocardiography was re-examined in 336 patients with HFrEF 6 months after discharge. Compared with the level on admission, the LVEF markedly improved at the sixth month after taking Sacubitril-Valsartan (48.49\% vs. 39.07\%, $\mathrm{P}<0.01$ ), while the LVEDD (54.70 vs. $59.97 \mathrm{~mm}, \mathrm{P}<0.01$ ), LVESD (40.59 vs. 47.59 mm, $\mathrm{P}<0.01$ ), LAD (48.59 vs. 52.45 $\mathrm{mm}, \mathrm{P}<0.01)$ and LVMI $\left(105.16\right.$ vs. $\left.125.20 \mathrm{~g} / \mathrm{m}^{2}, \mathrm{P}<0.01\right)$ decreased significantly. These results were shown in Table 2.

Among all participants, 135 cases underwent echocardiographic examination at the third and sixth month. The results of variance analysis based on repeated

Table 2 Primary and secondary outcomes of the general population $(\mathrm{n}=336)$

\begin{tabular}{lccc}
\hline Indicators & On admission & The 6th month & P value \\
\hline LVEF $(\%)$ & $39.07 \pm 10.63$ & $48.49 \pm 12.31$ & $<0.01^{*}$ \\
LVEDD $(\mathrm{mm})$ & $59.97 \pm 9.17$ & $54.70 \pm 9.59$ & $<0.01^{*}$ \\
LVESD $(\mathrm{mm})$ & $47.59 \pm 10.53$ & $40.21 \pm 11.16$ & $<0.01^{*}$ \\
LAD $(\mathrm{mm})$ & $52.45 \pm 12.54$ & $48.59 \pm 13.50$ & $<0.01^{*}$ \\
LVPW $(\mathrm{mm})$ & $8.82 \pm 1.64$ & $8.76 \pm 1.58$ & 0.67 \\
IVS $(\mathrm{mm})$ & $9.72 \pm 2.19$ & $9.46 \pm 2.17$ & 0.11 \\
LVMI $\left(\mathrm{g} / \mathrm{m}^{2}\right)$ & $125.20 \pm 39.62$ & $105.16 \pm 36.82$ & $<0.01^{*}$ \\
\hline
\end{tabular}

Data are presented as mean \pm SD or number (\%) of subjects.

*, $P<0.01$. LVEF, left ventricular ejection fraction; LVEDD, left ventricular end diastolic diameter; LVESD, left ventricular end systolic dimension; LAD, left atrium diameter; LVPW, left ventricular posterior wall; IVS, interventricular septum; LVMI, left ventricular mass index. measurement data showed significant differences in LVEF, LVEDD, LVESD, LAD and LVMI at baseline, the third month and the sixth month $(\mathrm{P}<0.05)$ (Table 3). After adjusting for confounding factors by using the mixed effect model, the LVEF at the third and the sixth month after taking Sacubitril-Valsartan increased by $8.37 \%(\mathrm{P}<0.01)$ and $10.49 \%(\mathrm{P}<0.01)$ compared with the level on admission. This data suggested that LVEF was improving as the treatment progressed, and the most obvious increase was in the early stages of treatment. The LVEDD, LVESD, LAD and LVMI all decreased significantly and similar variation tendency were observed. These results were shown in Table 4.

\section{Follow-up of the subpopulation}

A total of 223 patients were diagnosed as HFrEF on admission. In this subgroup, compared with the level at baseline, the LVEF increased significantly by the sixth month $(44.89 \%$ vs. $32.88 \%, \mathrm{P}<0.01)$, while the LVEDD (56.95 vs. $63.12 \mathrm{~mm}, \mathrm{P}<0.01)$, LVESD (42.96 vs. $51.77 \mathrm{~mm}$, $\mathrm{P}<0.01)$, LAD (47.94 vs. $52.36 \mathrm{~mm}, \mathrm{P}<0.01)$ and LVMI (110.05 vs. $\left.130.68 \mathrm{~g} / \mathrm{m}^{2}, \mathrm{P}<0.01\right)$ decreased significantly. These results were shown in Table 5.

Subgroup analysis was conducted according to gender. It was found that the LVEF of male HFrEF patients increased significantly after taking Sacubitril-Valsartan for 6 months (48.23\% vs. 39.01\%, $\mathrm{P}<0.01$ ), while the LVEDD, LEVSD, LAD and LVMI decreased significantly (all $\mathrm{P}<0.01$ ). In the subgroup of female patients, significant changes were observed only in LVEF, LVEDD, LVESD and LVMI values, while no significant difference occurred in LAD value. These results were shown in Table 6 .

Table 3 Primary outcomes in patients underwent echocardiography at the third and sixth month $(\mathrm{n}=135)$

\begin{tabular}{lcccc}
\hline Indicators & On admission & The 3rd month & The 6th month & P value \\
\hline LVEF $(\%)$ & $38.40 \pm 10.29$ & $46.91 \pm 10.84$ & $49.25 \pm 12.04$ & $<0.01^{*}$ \\
LVEDD $(\mathrm{mm})$ & $58.74 \pm 8.24$ & $54.01 \pm 7.41$ & $53.65 \pm 8.70$ & $<0.01^{*}$ \\
LVESD $(\mathrm{mm})$ & $46.51 \pm 10.10$ & $39.99 \pm 9.85$ & $39.31 \pm 10.39$ & $0.01^{* *}$ \\
LAD $(\mathrm{mm})$ & $52.16 \pm 11.64$ & $48.15 \pm 10.89$ & $47.54 \pm 13.54$ & 0.19 \\
LVPW $(\mathrm{mm})$ & $8.40 \pm 1.44$ & $8.81 \pm 1.65$ & $8.72 \pm 1.70$ & 0.26 \\
IVS $(\mathrm{mm})$ & $9.31 \pm 1.84$ & $8.49 \pm 1.73$ & $9.71 \pm 2.06$ & $104.74 \pm 35.65$ \\
LVMI $\left(\mathrm{g} / \mathrm{m}^{2}\right)$ & $114.63 \pm 29.43$ & $104.15 \pm 26.51$ & $0.01^{* \star}$ & \\
\hline
\end{tabular}

Data are presented as mean $\pm \mathrm{SD}$ or number (\%) of subjects. ${ }^{*}, \mathrm{P}<0.01 ;{ }^{\star \star}, \mathrm{P}<0.05 . \mathrm{P}$ value comes from variance analysis. $\mathrm{LVEF}$, left ventricular ejection fraction; LVEDD, left ventricular end diastolic diameter; LVESD, left ventricular end systolic dimension; LAD, left atrium diameter; LVPW, left ventricular posterior wall; IVS, interventricular septum; LVMI, left ventricular mass index. 
Table 4 Primary outcomes in patients underwent echocardiography at the third and sixth month $(\mathrm{n}=135)$

\begin{tabular}{|c|c|c|c|c|c|}
\hline \multirow{2}{*}{ Indicators } & \multirow{2}{*}{$\begin{array}{c}\text { On } \\
\text { admission }\end{array}$} & \multicolumn{2}{|c|}{ The 3rd month } & \multicolumn{2}{|c|}{ The 6th month } \\
\hline & & $\beta$ & $P$ value & $\beta$ & $P$ value \\
\hline LVEF (\%) & Ref & 8.37 & $<0.01^{*}$ & 10.49 & $<0.01^{*}$ \\
\hline LVEDD (mm) & Ref & -4.73 & $<0.01^{*}$ & -4.94 & $<0.01^{*}$ \\
\hline LVESD (mm) & Ref & -6.57 & $<0.01^{\star}$ & -6.96 & $<0.01^{*}$ \\
\hline LAD (mm) & Ref & -4.05 & $0.02^{\star \star}$ & -4.63 & $0.01^{\star *}$ \\
\hline LVPW (mm) & Ref & 0.41 & 0.08 & 0.30 & 0.21 \\
\hline IVS (mm) & Ref & 0.19 & 0.45 & 0.39 & 0.12 \\
\hline $\operatorname{LVMI}\left(\mathrm{g} / \mathrm{m}^{2}\right)$ & Ref & 10.43 & $0.01^{\star \star}$ & -9.59 & $0.01^{\star *}$ \\
\hline
\end{tabular}

Data are presented as mean \pm SD or number (\%) of subjects. ${ }^{*}, \mathrm{P}<0.01 ;{ }^{* *}, \mathrm{P}<0.05$. The value of $\mathrm{P}$ comes from the mixed effect model, and the adjusted confounding factors include age, sex, height, weight, SBP, DBP, HR, BNP, NYHA symptom severity class, medical history, previous medication, creatinine and eGFR. LVEF, left ventricular ejection fraction; LVEDD, left ventricular end diastolic diameter; LVESD, left ventricular end systolic dimension; LAD, left atrium diameter; LVPW, left ventricular posterior wall; IVS, interventricular septum; LVMI, left ventricular mass index.
Table 5 Primary outcomes in the subgroups of patients diagnosed with HFrEF on admission ( $\mathrm{n}=223)$

\begin{tabular}{lccc}
\hline Indicators & On admission & The 6th month & P value \\
\hline LVEF $(\%)$ & $32.88 \pm 5.48$ & $44.89 \pm 12.24$ & $<0.01^{*}$ \\
LVEDD $(\mathrm{mm})$ & $63.12 \pm 8.65$ & $56.95 \pm 9.97$ & $<0.01^{*}$ \\
LVESD $(\mathrm{mm})$ & $51.77 \pm 9.51$ & $42.96 \pm 11.69$ & $<0.01^{*}$ \\
LAD $(\mathrm{mm})$ & $52.36 \pm 13.29$ & $47.94 \pm 13.61$ & $0.001^{*}$ \\
LVPW $(\mathrm{mm})$ & $8.63 \pm 1.66$ & $8.68 \pm 1.51$ & 0.75 \\
IV S $(\mathrm{mm})$ & $9.20 \pm 2.08$ & $9.15 \pm 2.13$ & 0.82 \\
LVMI $\left(\mathrm{g} / \mathrm{m}^{2}\right)$ & $130.68 \pm 41.69$ & $110.05 \pm 39.67$ & $<0.01^{*}$ \\
\hline
\end{tabular}

Data are presented as mean \pm SD or number (\%) of subjects. ${ }^{*}, \mathrm{P}<0.01$. LVEF, left ventricular ejection fraction; LVEDD, left ventricular end diastolic diameter; LVESD, left ventricular end systolic dimension; LAD, left atrium diameter; LVPW, left ventricular posterior wall; IVS, interventricular septum; LVMI, left ventricular mass index.

Table 6 Primary and secondary outcomes in the gender subgroups

\begin{tabular}{|c|c|c|c|c|c|c|}
\hline Indicators & \multicolumn{3}{|c|}{ Female $(n=68)$} & \multicolumn{3}{|c|}{ Male $(n=268)$} \\
\hline LVEF (\%) & $39.32 \pm 9.33$ & $49.50 \pm 10.78$ & $<0.01^{\star}$ & $39.01 \pm 10.96$ & $48.23 \pm 12.70$ & $<0.01^{*}$ \\
\hline LVEDD (mm) & $55.18 \pm 7.68$ & $49.35 \pm 7.24$ & $<0.01^{*}$ & $61.19 \pm 9.15$ & $56.05 \pm 9.66$ & $<0.01^{*}$ \\
\hline LVESD (mm) & $43.71 \pm 8.86$ & $35.24 \pm 6.72$ & $<0.01^{\star}$ & $48.57 \pm 10.72$ & $41.48 \pm 11.71$ & $<0.01^{*}$ \\
\hline LVPW (mm) & $8.81 \pm 1.02$ & $8.52 \pm 1.34$ & 0.35 & $8.82 \pm 1.76$ & $8.81 \pm 1.63$ & 0.98 \\
\hline IVS (mm) & $9.39 \pm 1.61$ & $8.54 \pm 1.81$ & $0.03^{\star \star}$ & $9.81 \pm 2.31$ & $9.69 \pm 2.20$ & 0.52 \\
\hline LVMI $\left(\mathrm{g} / \mathrm{m}^{2}\right)$ & $122.02 \pm 30.09$ & $93.50 \pm 27.69$ & $<0.01^{\star}$ & $126.01 \pm 41.75$ & $108.11 \pm 38.33$ & $<0.01^{*}$ \\
\hline
\end{tabular}

Data are presented as mean \pm SD or number (\%) of subjects. ${ }^{*}, \mathrm{P}<0.01$; ${ }^{*}, \mathrm{P}<0.05$. LVEF, left ventricular ejection fraction; LVEDD, left ventricular end diastolic diameter; LVESD, left ventricular end systolic dimension; LAD, left atrium diameter; LVPW, left ventricular posterior wall; IVS, interventricular septum; LVMI, left ventricular mass index.

Considering the different cardiac functional classes, it was found that the LVEF of patients with NYHA II increased significantly after taking Sacubitril-Valsartan for 6 months (48.88\% vs. $40.89 \%, \mathrm{P}<0.01$ ), while the LVEDD, LEVSD, LAD and LVMI decreased significantly. After treatment, the LVEF of patients with NYHA III increased significantly ( $47.53 \%$ vs. $35.89 \%, \mathrm{P}<0.01)$, LVEDD, LEVSD, LAD and LVMI decreased significantly. However, in NYHA IV patients, only the LVEF increased marginally after treatment $(45.43 \%$ vs. $35.57 \%, \mathrm{P}=0.05)$, and the other indicators did not change significantly. These results were shown in Table 7. 
Table 7 Primary and secondary outcomes in subgroups with different cardiac function classes

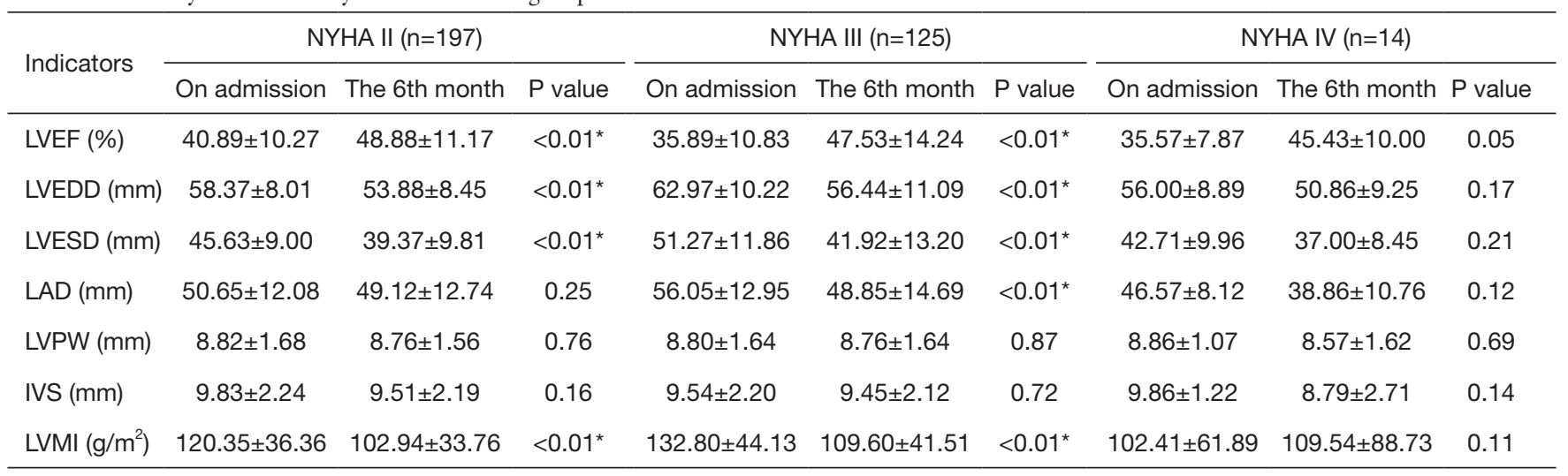

Data are presented as mean \pm SD or number (\%) of subjects. *, $\mathrm{P}<0.01$. LVEF, left ventricular ejection fraction; LVEDD, left ventricular end diastolic diameter; LVESD, left ventricular end systolic dimension; LAD, left atrium diameter; LVPW, left ventricular posterior wall; IVS, interventricular septum; LVMI, left ventricular mass index.

\section{Discussion}

HF leads to reduced activity tolerance and repeated hospitalizations, and seriously affects the quality of life, which is a major cause of death among the elderly. In recent years, great progress has been made in the field of $\mathrm{HF}$ treatment in China, and the overall mortality rate of HF has decreased, but the morbidity, mortality and rehospitalization rate of the elderly patients are still high $(8,9)$. The study found that the mortality rate of elderly patients was over $7.8 \%$ (10). The rapid increase of the incidence of elderly patients is related to senility, the increase of comorbidities such as hypertension, coronary heart disease, diabetes, and prolonged survival due to the improvement of medical environment. Thus, we should focus on elderly people.

In this retrospective study, 336 elderly patients with HFrEF were enrolled to observe the changes of cardiac function and structure after taking Sacubitril-Valsartan for 6 months. It was found that in the overall population, the LVEF increased significantly by the sixth month after taking Sacubitril-Valsartan, while the LVEDD, LEVSD, LAD and LVMI decreased significantly. These results were confirmed by the previous three studies (11-13). In subgroup analysis, the improvement of heart function in male and NHYA II and III subgroups was more substantial than in women and NYHA IV subgroups.

HFrEF is a serious and terminal stage of various cardiovascular diseases with high hospitalization and mortality rate (14-16). Its outcome will be cardiac dilatation and obvious function decrease (17). The progression of HFrEF is always accompanied by changes in cardiac function and structure; on the contrary, the improvement of cardiac structure will inevitably lead to the improved condition of patients with HF. Remodeling of the myocardium is central to the progression of HFrEF (18-20). It is affected by myocardial injury, hemodynamic changes or neurohormonal activation. In general, remodeling consists of the changes of cardiac geometry, function, or both, which are reflected by LVEF and increased left ventricular (LV) volumes. Cardiac remodeling is associated with risk of cardiovascular events, including death and hospitalization for HF, which is an important target for HFrEF therapy. In studies of guideline-directed medical therapies for HFrEF, taking $\beta$ blockers, ACEI/ARB/ARNI and spironolactone have shown to improve LVEF and/or decrease left ventricular volume, as well as improve the clinical outcomes (21-25). Januzzi et al. (26) found that the cardiac volume and function of patients with HFrEF were significantly improved after taking Sacubitril-Valsartan for 12 months, and the observation of reverse cardiac remodeling may provide a mechanistic explanation for drug treatment of such patients. In the research from Desai et al. (27), a similar conclusion was obtained. Although the duration of followup and sample size needs to be improved, the overall results of our study are basically consistent with theirs.

In this study, two sets of data were obtained in some participants who underwent echocardiographic examination at the third and sixth month. This data suggested that the measured outcomes were improving as time progressed, but the most obvious increase was in the early stage after taking medicine. The result was partial consistency with the find of Velazquez et al. (28). Although the included 
patients were different, they showed that the reduction in the NT-proBNP concentration was significantly greater at the early phase. In the subgroups of patients diagnosed with HFrEF on admission, the results were basically consistent with those in overall population. Further subgroups were analyzed according to gender and NYHA class. In the gender subgroup, we found that male patients benefited more than female patients. These results seemed to be inconsistent with the research of McMurray et al. (29), in which female patients accounted for the majority of the population, and these patients were all diagnosed as $\mathrm{HF}$ with preserved ejection fraction. Moreover, patients in NHYA II and III subgroups benefited more than in other NHYA classes. The result was different from the data of Velazquez et al. (28). It should be noted that the sample size of NHYA IV patients (14 cases) was the smallest, accounting for only $4.2 \%$ of the total participants, which may lead to the decrease of statistical efficiency. However, we still found that, the LVEF increased marginally (45.43\% vs. $35.57 \%$, $\mathrm{P}=0.05)$, which suggested that taking Sacubitril-Valsartan for 6 months may be effective for the kind of patient. Thus, the increase in sample size of NHYA IV in future would be helpful to obtain the same results as NHYA II and III subgroups. In brief, these results suggested that gender and cardiac function grade may influence the therapeutic effect of Sacubitril-Valsartan in elderly patients with HFrEF.

There are also some deficiencies in this study. Firstly, the sample size of female and NYHA IV subgroups are smaller than other subgroups. Herein, studies with a larger sample size and a more rigorous design are needed in the future. Secondly, due to the lack of information about the clinical endpoints of patients in this study, it is not possible to assess how changes in cardiac function and structure lead to direct clinical benefits.

In conclusion, Sacubitril-Valsartan can improve the cardiac function and structure of elderly patients with HFrEF. More improvement of cardiac is observed in men and NYHA II and III subgroups, although further studies with larger sample size are needed.

\section{Acknowledgments}

We would like to thank Eric Nicholas Werbitsky for his help in polishing our paper and all other people who make contributions to this work.

Funding: This work was supported by the Beijing Natural Science Foundation (7204252); and the Beijing Hospitals Authority Youth Programme (QML20200603).

\section{Footnote}

Reporting Checklist: The authors have completed the STROBE reporting checklist. Available at https://dx.doi. org/10.21037/cdt-21-355

Data Sharing Statement: Available at https://dx.doi. org/10.21037/cdt-21-355

Conflicts of Interest: All authors have completed the ICMJE uniform disclosure form (available at https://dx.doi. org/10.21037/cdt-21-355). The authors have no conflicts of interest to declare.

Etbical Statement: The authors are accountable for all aspects of the work in ensuring that questions related to the accuracy or integrity of any part of the work are appropriately investigated and resolved. The present study was conducted in accordance with the Declaration of Helsinki (as revised in 2013) and was approved by the ethics committee of Beijing Anzhen Hospital (No. 2021090X), and individual consent for this retrospective analysis was waived.

Open Access Statement: This is an Open Access article distributed in accordance with the Creative Commons Attribution-NonCommercial-NoDerivs 4.0 International License (CC BY-NC-ND 4.0), which permits the noncommercial replication and distribution of the article with the strict proviso that no changes or edits are made and the original work is properly cited (including links to both the formal publication through the relevant DOI and the license). See: https://creativecommons.org/licenses/by-nc-nd/4.0/.

\section{References}

1. The Writing Committee of the Report on Cardiovascular Health and Diseases in China. Report on Cardiovascular Health and Diseases in China 2019: An Updated Summary. Zhongguo Xun Huan Za Zhi (Chinese) 2020;35:833-54.

2. Heart Failure Group of Cardiovascular Branch of Chinese Medical Association. Guidelines for Diagnosis and Treatment of Heart Failure in China 2018. Zhonghua Xin Xue Guan Bing Za Zhi (Chinese) 2018;46:760-89.

3. Rubattu S, Sciarretta S, Volpe M. Atrial natriuretic peptide gene variants and circulating levels: implications in cardiovascular diseases. Clin Sci (Lond) 2014;127:1-13.

4. Boerrigter G, Burnett JC Jr. Recent advances in natriuretic peptides in congestive heart failure. Expert Opin Investig 
Drugs 2004;13:643-52.

5. McMurray JJ, Packer M, Desai AS, et al. Angiotensinneprilysin inhibition versus enalapril in heart failure. $\mathrm{N}$ Engl J Med 2014;371:993-1004.

6. Solomon SD, Zile M, Pieske B, et al. The angiotensin receptor neprilysin inhibitor LCZ696 in heart failure with preserved ejection fraction: a phase 2 double-blind randomised controlled trial. Lancet 2012;380:1387-95.

7. Devereux RB, Alonso DR, Lutas EM, et al. Echocardiographic assessment of left ventricular hypertrophy: comparison to necropsy findings. Am J Cardiol 1986;57:450-8.

8. Gu DF, Huang GY, HeJ, et al. Investigation of prevalence and distributing feature of chronic heart failure in Chinese adult population. Zhonghua Xin Xue Guan Bing Za Zhi (Chinese) 2003;31:3-6.

9. Hao G, Wang X, Chen Z, et al. Prevalence of heart failure and left ventricular dysfunction in China: the China Hypertension Survey, 2012-2015. Eur J Heart Fail 2019;21:1329-37.

10. Pei ZY, Zhao YS, Li JY, et al. Fifteen-year evolving trends of etiology and prognosis in hospitalized patients with heart failure. Zhonghua Xin Xue Guan Bing Za Zhi 2011;39:434-9.

11. Almufleh A, Marbach J, Chih S, et al. Ejection fraction improvement and reverse remodeling achieved with Sacubitril/Valsartan in heart failure with reduced ejection fraction patients. Am J Cardiovasc Dis 2017;7:108-13.

12. Polito MV, Silverio A, Rispoli A, et al. Clinical and echocardiographic benefit of Sacubitril/Valsartan in a realworld population with HF with reduced ejection fraction. Sci Rep 2020;10:6665.

13. Moon MG, Hwang IC, Choi W, et al. Reverse remodelling by sacubitril/valsartan predicts the prognosis in heart failure with reduced ejection fraction. ESC Heart Fail 2021;8:2058-69.

14. Riedel O, Ohlmeier C, Enders D, et al. The contribution of comorbidities to mortality in hospitalized patients with heart failure. Clin Res Cardiol 2018;107:487-97.

15. Platz E, Jhund PS, Claggett BL, et al. Prevalence and prognostic importance of precipitating factors leading to heart failure hospitalization: recurrent hospitalizations and mortality. Eur J Heart Fail 2018;20:295-303.

16. Murphy SP, Ibrahim NE, Januzzi JL Jr. Heart Failure With Reduced Ejection Fraction: A Review. JAMA 2020;324:488-504.

17. Hartupee J, Mann DL. Neurohormonal activation in heart failure with reduced ejection fraction. Nat Rev Cardiol 2017;14:30-8.

18. Kemp CD, Conte JV. The pathophysiology of heart failure. Cardiovasc Pathol 2012;21:365-71.
19. Konstam MA, Kramer DG, Patel AR, et al. Left ventricular remodeling in heart failure: current concepts in clinical significance and assessment. JACC Cardiovasc Imaging 2011;4:98-108.

20. Udelson JE, Konstam MA. Ventricular remodeling fundamental to the progression (and regression) of heart failure. J Am Coll Cardiol 2011;57:1477-9.

21. Cao Y, Rui MJ, Guan X, et al. Irbesartan combined with metoprolol compared with single-drug in the treatment of elderly patients with severe heart failure: a network metaanalysis. Chinese Journal of Evidence-Based Medicine 2020;20:672-80.

22. Shah A, Gandhi D, Srivastava S, et al. Heart Failure: A Class Review of Pharmacotherapy. P T 2017;42:464-72.

23. Li H, Duan Y, Chen B, et al. New pharmacological treatments for heart failure with reduced ejection fraction (HFrEF): A Bayesian network meta-analysis. Medicine (Baltimore) 2020;99:e18341.

24. Gilstrap L, Austin A, Gladders B, et al. Beta Blockers After HFrEF Hospitalization Decrease 1-Year Mortality Across the Age Spectrum. Circulation 2019;140:A14853.

25. Abboud A, Januzzi JL. Reverse Cardiac Remodeling and ARNI Therapy. Curr Heart Fail Rep 2021;18:71-83.

26. Januzzi JL Jr, Prescott MF, Butler J, et al. Association of Change in N-Terminal Pro-B-Type Natriuretic Peptide Following Initiation of Sacubitril-Valsartan Treatment With Cardiac Structure and Function in Patients With Heart Failure With Reduced Ejection Fraction. JAMA 2019;322:1085-95.

27. Desai AS, Solomon SD, Shah AM, et al. Effect of Sacubitril-Valsartan vs Enalapril on Aortic Stiffness in Patients With Heart Failure and Reduced Ejection Fraction: A Randomized Clinical Trial. JAMA 2019;322:1077-84.

28. Velazquez EJ, Morrow DA, DeVore AD, et al. AngiotensinNeprilysin Inhibition in Acute Decompensated Heart Failure. N Engl J Med 2019;380:539-48.

29. McMurray JJV, Jackson AM, Lam CSP, et al. Effects of Sacubitril-Valsartan Versus Valsartan in Women Compared With Men With Heart Failure and Preserved Ejection Fraction: Insights From PARAGON-HF. Circulation 2020;141:338-51.

Cite this article as: $\mathrm{Gu}$ W, Xu C, Li Z, Li ZZ. Echocardiographic changes in elderly patients with heart failure with reduced ejection fraction after sacubitril-valsartan treatment. Cardiovasc Diagn Ther 2021;11(5):1093-1100. doi: 10.21037/cdt-21-355 